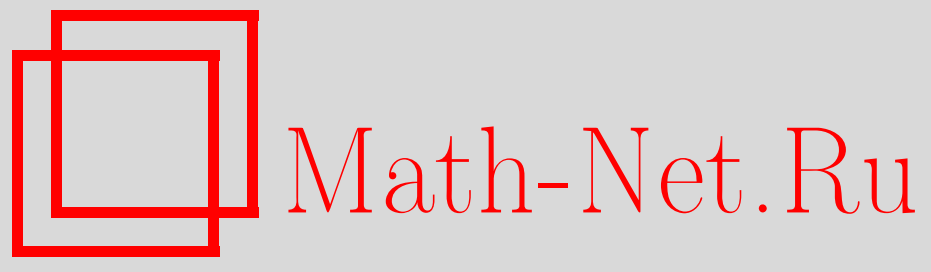

А. В. Устинов, К трехмерной теореме Валена, Матем. заметки, 2014, том 95, выпуск 1, 154-156

DOI: https://doi.org/10.4213/mzm10110

Использование Общероссийского математического портала Math-Net.Ru подразумевает, что вы прочитали и согласны с пользовательским соглашением http://www . mathnet.ru/rus/agreement

Параметры загрузки:

IP : 35.173 .219 .12

26 апреля 2023 г., 16:08:02 


\section{К трехмерной теореме Валена}

\section{А. В. Устинов}

1. Введение. Пусть $\gamma^{(1)}, \ldots, \gamma^{(s)}$ - базисные узлы полной решетки

$$
\Gamma=\left\{m_{1} \gamma^{(1)}+\cdots+m_{s} \gamma^{(s)}: m_{1}, \ldots, m_{s} \in \mathbb{Z}\right\} \subset \mathbb{R}^{s} .
$$

Решетка всегда будет считаться решеткой «общего положения»: координатные гиперплоскости не содержат узлов решетки, отличных от начала координат. (Относительно общего случая см. [1].) Обозначим через $G_{s}$ конечную группу, действующую на $G L_{s}(\mathbb{R})$, порожденную следующими элементарными преобразованиями: 1) изменение знаков у элементов строки или столбца; 2) перестановка строк или столбцов. Две матрицы назовем эквивалентными, если одна может быть получена из другой с помощью некоторого преобразования из $G_{s}$.

Предположим, что $\left\{\gamma^{(1)}, \gamma^{(2)}\right\}$ - базис двумерной решетки $Г$, для которого не существует ненулевых узлов $\gamma=\left(\gamma_{1}, \gamma_{2}\right) \in \Gamma$ таких, что

$$
\left|\gamma_{1}\right|<\max \left\{\left|\gamma_{1}^{(1)}\right|,\left|\gamma_{1}^{(2)}\right|\right\}, \quad\left|\gamma_{2}\right|<\max \left\{\left|\gamma_{2}^{(1)}\right|,\left|\gamma_{2}^{(2)}\right|\right\} .
$$

Вороной доказал (см. [2]), что это возможно тогда и только тогда, когда для некоторого преобразования $\Phi \in G_{2}$

$$
\Phi\left(\begin{array}{ll}
\gamma_{1}^{(1)} & \gamma_{1}^{(2)} \\
\gamma_{2}^{(1)} & \gamma_{2}^{(2)}
\end{array}\right)=\left(\begin{array}{cc}
a_{1} & -b_{1} \\
a_{2} & b_{2}
\end{array}\right), \quad \text { где } \quad 0 \leqslant b_{1} \leqslant a_{1}, \quad 0 \leqslant a_{2} \leqslant b_{2} .
$$

Базис решетки называется базисом Вороного, если соответствующая ему матрица эквивалентна матрице вида $\left(\begin{array}{cc}a_{1} & -b_{1} \\ a_{2} & b_{2}\end{array}\right)$.

В статье [3] отмечено, что теорема Валена о приближении чисел подходящими дробями (см. [4], [5]) в терминах решеток имеет следующую интерпретацию: для любого базиса Вороного $\left\{\gamma^{(1)}, \gamma^{(2)}\right\}$

$$
\min \left\{\left|\gamma_{1}^{(1)} \gamma_{2}^{(1)}\right|,\left|\gamma_{1}^{(2)} \gamma_{2}^{(2)}\right|\right\} \leqslant \frac{1}{2} \operatorname{det} \Gamma
$$

В той же статье было предложено уточнение теоремы Валена

$$
\left|\gamma_{1}^{(1)} \gamma_{2}^{(1)}\right|+\left|\gamma_{1}^{(2)} \gamma_{2}^{(2)}\right| \leqslant \operatorname{det} \Gamma
$$

следующее из неравенства

$$
a_{1} b_{2}+a_{2} b_{1}-a_{1} a_{2}-b_{1} b_{2}=\left(a_{1}-b_{1}\right)\left(b_{2}-a_{2}\right) \geqslant 0 .
$$

В работе [3] Авдеевой и Быковским был доказан аналог неравенства (1) для базисов Минковского (трехмерных аналогов базисов Вороного). Ниже предлагается более простое и короткое доказательство теоремы Авдеевой и Быковского. Кроме того их результат распространяется на произвольные минимальные системы из трех векторов (см. определения ниже).

Работа выполнена при поддержке Российского фонда фундаментальных исследований (грант № 11-01-00628-а), программы "Ведущие научные школы” (грант № НШ-1922.2012.1) и фонда «Династия».

DOI: $10.4213 / \mathrm{mzm} 10110$

(C) А. В. Устинов, 2014 
2. Минимальные системы и базисы Минковского. Для непустого точечного множества $T \subset \mathbb{R}^{3}$ положим

$$
\begin{gathered}
|T|_{i}=\max \left\{\left|x_{i}\right|: x=\left(x_{1}, x_{2}, x_{3}\right) \in T\right\}, \quad i=1,2,3, \\
\Pi(T)=\left[-|T|_{1},|T|_{1}\right] \times\left[-|T|_{2},|T|_{2}\right] \times\left[-|T|_{3},|T|_{3}\right] .
\end{gathered}
$$

Пусть $Г$ - полная решетка в $\mathbb{R}^{3}$. Системой узлов порядка $t$ решетки $Г$ назовем любой конечный набор ненулевых узлов $Г$ вида $\left(\gamma^{(1)}, \ldots, \gamma^{(t)}\right)$, где $\gamma^{(i)} \neq \pm \gamma^{(j)}, 1 \leqslant i<j \leqslant t$. Система $S$ решетки Г называется минимальной, если не существует ненулевых узлов $\gamma \in \Gamma$, лежащих строго внутри $\Pi(S)$.

Минковским доказано (см. [6], [7; статья 109]), что всякая минимальная система из трех векторов $\left(\gamma^{(1)}, \gamma^{(2)}, \gamma^{(3)}\right)$ либо вырождена (и тогда для некоторой комбинации знаков $\gamma^{(1)} \pm \gamma^{(2)} \pm \gamma^{(3)}=0$ ), либо образует базис решетки (базис Минковского). С помощью элементарных преобразований матрица любого базиса Минковского может быть приведена к одной из двух канонических форм (см. [6], [7], а также [8]-[10])

$$
\left(\begin{array}{ccc}
a_{1} & b_{1} & -c_{1} \\
-a_{2} & b_{2} & c_{2} \\
a_{3} & -b_{3} & c_{3}
\end{array}\right), \quad\left(\begin{array}{ccc}
a_{1} & b_{1} & c_{1} \\
-a_{2} & b_{2} & c_{2} \\
a_{3} & -b_{3} & c_{3}
\end{array}\right)
$$

с неотрицательными $a_{i}, b_{i}, c_{i}, i=1,2,3$, удовлетворяющими условиям:

1) $\max \left\{b_{1}, c_{1}\right\} \leqslant a_{1}, \max \left\{a_{2}, c_{2}\right\} \leqslant b_{2}, \max \left\{a_{3}, b_{3}\right\} \leqslant c_{3}$;

2) для матриц первого типа выполняется по крайней мере одно из неравенств $c_{1} \leqslant b_{1}$, $a_{2} \leqslant c_{2}, b_{3} \leqslant c_{3} ;$

$3)$ для матриц второго типа выполняются неравенство $a_{2}+c_{2} \geqslant b_{2}$ и по крайней мере одно из неравенств $a_{3} \leqslant b_{3}, c_{1} \leqslant b_{1}$.

При этом за счет переобозначения координат и векторов можно добиться того, чтобы из трех (для матриц первого типа) или двух (для матриц второго типа) альтернативных неравенств выполнялось неравенство $c_{1} \leqslant b_{1}$.

3. Трехмерная теорема Валена. Предыдущие результаты, связанные с трехмерной теоремой Валена, а также некоторые ее уточнения могут быть найдены в [10]-[12].

Теорема 1 (Авдеева, Быковский, 2006). Пусть узль $\gamma^{(1)}, \gamma^{(2)}, \gamma^{(3)}$ составляют базис Минковского рещетки Г. Тогда

$$
\left|\gamma_{1}^{(1)} \gamma_{2}^{(1)} \gamma_{3}^{(1)}\right|+\left|\gamma_{1}^{(2)} \gamma_{2}^{(2)} \gamma_{3}^{(2)}\right|+\left|\gamma_{1}^{(3)} \gamma_{2}^{(3)} \gamma_{3}^{(3)}\right| \leqslant \operatorname{det} \Gamma .
$$

ДокАЗАтЕльство. Для матрицы $M$, столбцами которой являются векторы $\gamma^{(1)}, \gamma^{(2)}$, $\gamma^{(3)}$, докажем, что $\Delta(M) \geqslant 0$, где

$$
\Delta(M)=\operatorname{det} M-\left|\gamma_{1}^{(1)} \gamma_{2}^{(1)} \gamma_{3}^{(1)}\right|-\left|\gamma_{1}^{(2)} \gamma_{2}^{(2)} \gamma_{3}^{(2)}\right|-\left|\gamma_{1}^{(3)} \gamma_{2}^{(3)} \gamma_{3}^{(3)}\right|
$$

Под действием преобразований из группы $G_{3}$ величина $\Delta(M)$ не меняется. Поэтому оценку $\Delta(M) \geqslant 0$ достаточно доказать для базисов, матрицы которых имеют вид (2) и удовлетворяют неравенству $c_{1} \leqslant b_{1} \leqslant a_{1}$.

Так как $b_{2} c_{3} \geqslant a_{2} a_{3}$, то коэффициент при $a_{1}$ в разложении $(\varepsilon=-1$ для матриц первого типа и $\varepsilon=1$ для матриц второго типа)

$$
\Delta(M)=a_{1}\left(b_{2} c_{3}+c_{2} b_{3}-a_{2} a_{3}\right)+b_{1}\left(a_{2} c_{3}+c_{2} a_{3}-b_{2} b_{3}\right)+\varepsilon c_{1}\left(a_{2} b_{3}-b_{2} a_{3}\right)-c_{1} c_{2} c_{3},
$$

неотрицателен, поэтому неравенство $\Delta(M) \geqslant 0$ достаточно проверить при $a_{1}=b_{1}$. В этом случае

$$
\Delta(M)=b_{1}\left(b_{2}\left(c_{3}-b_{3}\right)+a_{2}\left(c_{3}-a_{3}\right)+c_{2}\left(a_{3}+b_{3}\right)\right)+\varepsilon c_{1}\left(a_{2} b_{3}-b_{2} a_{3}\right)-c_{1} c_{2} c_{3},
$$


и коэффициент при $b_{1}$ неотрицателен. Значит, достаточно ограничиться случаем, когда $a_{1}=b_{1}=c_{1}$. Для матриц первого типа $(\varepsilon=-1)$ и

$$
\Delta(M)=c_{1}\left(a_{3}\left(b_{2}-a_{2}\right)+a_{2}\left(c_{3}-b_{3}\right)+\left(b_{2}-c_{2}\right)\left(c_{3}-b_{3}\right)+a_{3} c_{2}\right) \geqslant 0,
$$

поскольку все слагаемые неотрицательны. Для матриц второго типа $(\varepsilon=1)$ и

$$
\Delta(M)=c_{1}\left(\left(b_{2}-c_{2}\right)\left(c_{3}-a_{3}\right)+a_{2}\left(c_{3}-a_{3}\right)+b_{3}\left(a_{2}+c_{2}-b_{2}\right)\right) \geqslant 0,
$$

поскольку выполняется дополнительное условие $a_{2}+c_{2} \geqslant b_{2}$.

4. Обобщение трехмерной теоремы Валена. Результат теоремы 1 допускает следующее уточнение.

Теорема 2. Неравенство (3) выполняется для произволъной минимальной системы из трех векторов $\gamma^{(1)}, \gamma^{(2)}, \gamma^{(3)}$.

ДокАЗАТЕльство. Учитывая теорему 1, доказательство достаточно провести для вырожденной минимальной тройки. Предположим, что $\left(\gamma^{(1)}, \gamma^{(2)}, \gamma^{(3)}\right)$ - вырожденная система из трех векторов, а их знаки выбраны так, что $\gamma^{(1)}+\gamma^{(2)}+\gamma^{(3)}=0$. Тогда матрица вырожденной минимальной тройки путем элементарных преобразований может быть приведена к виду (см. [7; статья 109])

$$
\left(\begin{array}{ccc}
a_{1} & -b_{1} & -c_{1} \\
-a_{2} & b_{2} & -c_{2} \\
-a_{3} & -b_{3} & c_{3}
\end{array}\right)=\left(\begin{array}{ccc}
b_{1}+c_{1} & -b_{1} & -c_{1} \\
-a_{2} & a_{2}+c_{2} & -c_{2} \\
-a_{3} & -b_{3} & a_{3}+b_{3}
\end{array}\right)
$$

с неотрицательными $a_{i}, b_{i}, c_{i}, i=1,2,3$. Параллелепипед $\left[-a_{1}, a_{1}\right] \times\left[-b_{2}, b_{2}\right] \times\left[-c_{3}, c_{3}\right]$ не содержит внутри себя точек решетки $\Gamma$, отличных от начала координат. По теореме Минковского о выпуклом теле $\operatorname{det} \Gamma \geqslant a_{1} b_{2} c_{3}$. Следовательно,

$$
\begin{aligned}
\left|\gamma_{1}^{(1)} \gamma_{2}^{(1)} \gamma_{3}^{(1)}\right|+\left|\gamma_{1}^{(2)} \gamma_{2}^{(2)} \gamma_{3}^{(2)}\right|+\left|\gamma_{1}^{(3)} \gamma_{2}^{(3)} \gamma_{3}^{(3)}\right| & =\left(b_{1}+c_{1}\right) a_{2} a_{3}+\left(a_{2}+c_{2}\right) b_{1} b_{3}+\left(a_{3}+b_{3}\right) c_{1} c_{2} \\
& \leqslant\left(b_{1}+c_{1}\right)\left(a_{2}+c_{2}\right)\left(a_{3}+b_{3}\right)=a_{1} b_{2} c_{3} \leqslant \operatorname{det} \Gamma
\end{aligned}
$$

\section{СПИСОК ЦИТИРОВАННОЙ ЛИТЕРАТУРЫ}

[1] А.В. Устинов, Математика и информатика, 1, K 75-летию со дня рождения Анатолия Алексеевича Карацубы, Совр. пробл. матем., 16, МИАН, М., 2012, 103-128. [2] Г. Ф. Вороной, Собрание сочинений, Т. 1, Изд-во АН УССР, Киев, 1952. [3] М. О. Авдеева,В. А. Быковский, Матем. заметки, 79:2 (2006), 163-168. [4] K. Th. Vahlen, J. für Math., 115:3 (1895), 221-233. [5] А. Я. Хинчин, Цепные дроби, Наука, М., 1978. [6] H. Minkowski, Ann. Sci. École Norm. Sup. (3), 13 (1896), 41-60. [7] H. Hancock, Development of the Minkowski Geometry of Numbers, Vol. 1, 2, Dover Publ., 1964. [8] O. А. Горкуша, Матем. заметки, 69:3 (2001), 353-362. [9] В. А. Быковский, О. А. Горкуша, Матем. сб., 192:2 (2001), 57-66. [10] М. О. Авдеева, В. А. Быковский, Матем. сб., 194:7 (2003), 3-14. [11] В. А. Быковский, Матем. заметки, 66:1 (1999), 30-37. [12] С. Гассан, Чебышевский сб., 6:3 (2005), $51-84$.

\section{А. В. Устинов}

Поступило

Хабаровское отделение

08.08.2012

Института прикладной математики ДВО РАН

вй вариант

E-mail: ustinov.alexey@gmail.com

31.07 .2013 